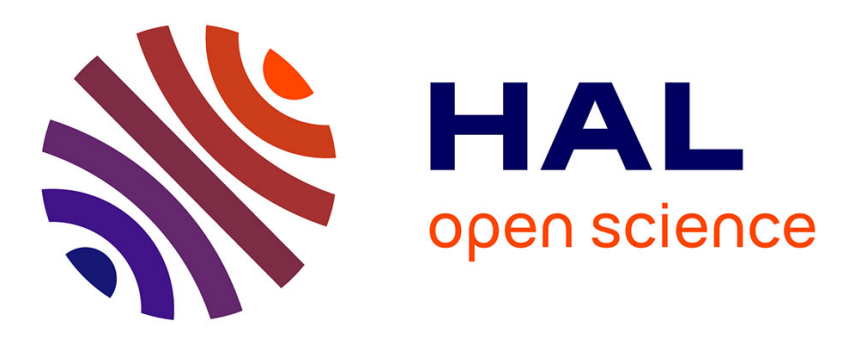

\title{
Indexer selon les voyelles: un ordre alphabétique inhabituel dans trois tables (exempla et distinctions) des Sermones ad status de Guibert de Tournai
}

Marjorie Burghart

\section{- To cite this version: \\ Marjorie Burghart. Indexer selon les voyelles: un ordre alphabétique inhabituel dans trois tables (exempla et distinctions) des Sermones ad status de Guibert de Tournai. Bibliotheque- Ecole des Chartes, 2008, 166 (2), pp.365-390. 10.3406/bec.2008.464687 . halshs-00449894}

\author{
HAL Id: halshs-00449894 \\ https://shs.hal.science/halshs-00449894
}

Submitted on 30 Aug 2021

HAL is a multi-disciplinary open access archive for the deposit and dissemination of scientific research documents, whether they are published or not. The documents may come from teaching and research institutions in France or abroad, or from public or private research centers.
L'archive ouverte pluridisciplinaire $\mathbf{H A L}$, est destinée au dépôt et à la diffusion de documents scientifiques de niveau recherche, publiés ou non, émanant des établissements d'enseignement et de recherche français ou étrangers, des laboratoires publics ou privés. 


\title{
INDEXER SELON LES VOYELLES
}

\author{
UN ORDRE ALPHABÉTIQUE INHABITUEL \\ DANS TROIS TABLES (EXEMPLA ET DISTINCTIONS) \\ Des «Sermones ad status » De Guibert de Tournai
}

par

MARJORIE BURGHART

Les études de Richard et Mary Rouse ont permis de mesurer l'importance, à partir du XIII ${ }^{\mathrm{e}}$ siècle, des instruments de travail intellectuel tels que les tables analytiques, en particulier pour les œuvres liées à l'activité des prédicateurs ${ }^{1}$. La collection des Sermones ad status composée par Guibert de Tournai offre de ce point de vue un champ d'étude particulièrement intéressant par l'ampleur de sa tradition ${ }^{2}$ : plus du quart des témoins, en effet, comportent au moins un index analytique. Tous respectent un ordre alphabétique plus ou moins strict; mais dans deux manuscrits les index sont soumis à une autre logique de classement, rangeant les entrées en fonction de leur première voyelle sans prendre en compte les consonnes. Il s'agit d'un principe alphabétique rare et a priori déconcertant, qui, s'il n'a

1. Voir notamment: Richard Rouse, «La diffusion en Occident au XIII ${ }^{\mathrm{e}}$ siècle des outils de travail facilitant l'accès aux textes autoritatifs », dans L'enseignement en Islam et en Occident au Moyen Âge, Paris, 1977, p. 115-147; id. et Mary Rouse, Preachers, florilegia and sermons, Toronto, 1979 ; eid., « Statim invenire : schools, preachers, and new attitudes to the page », dans Renaissance and renewal in the twelfth century, éd Robert L. Benson et Giles Constable, Cambridge (Mass.), 1982, p. 201-225. Outre ces travaux, on peut mentionner plus récemment : Letizia Pellegrini, «Tabula super sermones : gli indici dei sermonari domenicani nei codici centro-italiani (sec. XIII-Xv)», dans Archivum Fratrum praedicatorum, t. 64, 1994, p. 119-144; ; ead., «Indici per predicare : le tavole nei manoscritti di sermoni fra XIII e XV secolo », dans « Fabula in tabula » : una storia degli indici dal manoscritto al testo elettronico, éd. Claudio Leonardi, Marcello Morelli et Francesco Santi, Spolète, 1995 (Quaderni di cultura mediolatina, 13), p. 135-143; Franco Morenzoni et Paolo Mariani, «Quoniam sicut dicit Ysidorus : les tables médiévales de la Légende dorée », dans De la sainteté à l'hagiographie : genèse et usage de la Légende dorée, éd. Barbara Fleith et F. Morenzoni, Genève, 2001 (Publications romanes et françaises, 229), p. 173-209.

2. J'ai pu à ce jour identifier 109 manuscrits transmettant la collection complète ou des sermons qui en sont extraits.

Marjorie Burghart, ingénieur d'études à l'École des hautes études en sciences sociales (pôle de Lyon) - UMR 5648 (Histoire et archéologie des mondes chrétiens et musulmans médiévaux), 18 quai Claude-Bernard,69007 Lyon. <marjorie.burghart@ehess.fr> 
guère retenu l'attention jusqu'à présent, mérite d'être signalé afin d'enrichir la connaissance des techniques mises en œuvre pour faciliter l'accès aux instruments du travail intellectuel, en l'occurrence une des grandes collections de sermons ad status produites au XIII ${ }^{\mathrm{e}}$ siècle ${ }^{3}$.

\section{Les « Sermones ad status » de Guibert de Tournai.}

1. La collection. - Guibert, franciscain originaire de Tournai, enseigna la théologie à Paris où il fut maître-régent au tournant des années 1260, après Bonaventure dont il était proche. De l'homme, nous savons peu de chose, et ce sont souvent ses œuvres, nombreuses et variées, qui fournissent quelques renseignements ${ }^{4}$. Il est notamment l'auteur d'une série de cent dix sermons modèles ad status ${ }^{5}$, c'est-à-dire composés non pas en fonction d'une occasion liturgique, mais pour des auditoires théoriques correspondant chacun à un status, un état de vie défini dans la société. La collection est présentée dans le prologue comme composée de quatre parties inégales : de diversis statibus et officiis, soit la série ad status au sens propre (quatrevingt-seize sermons), de preceptis divinis (deux sermons), de sacramentalibus et mysteriis (neuf sermons), et de penis et gaudiis (trois sermons) ${ }^{6}$. La collection ayant toujours circulé ainsi, et les tables d'index ne séparant pas la partie ad status proprement dite des autres, il paraît opportun de considérer l'œuvre dans son ensemble. Cette série était conçue comme une partie du maître-ouvrage de Guibert, une somme intitulée Rudimentum doctrine $^{7}$. Si les manuscrits qui nous sont parvenus témoignent d'une circula-

3. Je tiens à remercier vivement Nicole Bériou de ses conseils et remarques.

4. Sur Guibert de Tournai, voir : Baudouin d'Amsterdam, «Guibert de Tournai », dans Dictionnaire de spiritualité, Paris, 1932-..., t. VI, col. 1139-1146; L. Baudry, «Wibert de Tournai », dans Revue d'histoire franciscaine, t. 5, 1928, p. 23-61; Guibert de Tournai, De modo addiscendi, éd. Enrico Bonifacio, Turin, 1953; Benjamin de Troeyer et Léonide Mees, Bio-bibliographia Franciscana Neerlandica ante saeculum XVI, Nieuwkoop, 1974, t. 1, p. 15-43; Palémon Glorieux, Répertoire des maîtres en théologie de Paris au XIII siècle, Paris, 1933-1934. (Études de philosophie médiévale, 17-18); id., «Maîtres franciscains régents à Paris : mise au point », dans Recherches de théologie ancienne et médiévale, t. 18, 1951, p. 324-332 ; Guibert de Tournai, Tractatus de pace, éd. Ephrem Longpré, Grottaferrata, 1925 (Bibliotheca Franciscana ascetica medii aevi, 6).

5. La liste donnée par Johannes Baptist Schneyer, Repertorium der lateinischen Sermones des Mittelalters für die Zeit von 1150-1350, Münster, 1969-1990 (Beiträge zur Geschichte der Philosophie und Theologie des Mittelalters, 43), t. II, p. 299-307, établie sur une mauvaise édition du $\mathrm{XvI}^{\mathrm{e}}$ siècle, est incomplète et doit être corrigée par la tradition manuscrite. Le Repertorium sera cité ci-après par le sigle $R L S$.

6. Les titres sont cités ici d'après le prologue du manuscrit Paris, Bibl. nat. Fr., lat. 15943, fol. 1.

7. Pour une introduction au Rudimentum, voir : Servus Gieben, «Il Rudimentum doctrinae di Gilberto di Tournai, con l'edizione del suo registrum o tavola della materia », dans Bonaventuriana : miscellanea in onore di Jacques Guy Bougerol OFM, éd. Francisco de Asis Chavaro Blanco, Rome, 1988, t. II, p. 621-680. Cette œuvre reste inédite dans son ensemble, 
tion indépendante des sermons, à quelques exceptions près, en revanche le prologue de la collection tout comme la table générale des matières présente dans les manuscrits du Rudimentum indiquent clairement que les sermons font bien partie du plan général de l'œuvre. Le recueil des Sermones ad status, composé après 1261, est l'une des trois grandes collections de ce genre produites au XIII ${ }^{\mathrm{e}}$ siècle, avec les Sermones vulgares de Jacques de Vitry et la série de modèles ad status incluse par Humbert de Romans dans son De eruditione predicatorum ${ }^{8}$. Si les collections de Guibert de Tournai et d'Humbert de Romans, sans doute rédigées approximativement à la même période ${ }^{9}$, sont indépendantes, Guibert a en revanche largement emprunté aux Sermones vulgares composés entre 1228 et 1240 par Jacques de Vitry ${ }^{10}$. Ces emprunts, connus de longue date ${ }^{11}$, ont été étudiés en détail pour certains groupes de sermons ${ }^{12}$. Il faut souligner que les emprunts aux $\mathrm{Ser}$ mones vulgares ne sont pas omniprésents : certains sermons sont indépendants de ceux de Jacques de Vitry; et, loin de simplement compiler les passages pris à son prédécesseur, Guibert a «exploité sans vergogne, et sans servilité » ${ }^{13}$, la matière à sa disposition. Ainsi, il a non seulement

à l'exception de parties isolées. Signalons qu'Aleksander Horowski poursuit actuellement le projet d'une édition critique du Rudimentum doctrine.

8. Il est à déplorer qu'aucune de ces œuvres ne bénéficie d'une édition critique intégrale : pour chacune, seuls des sermons isolés ont été édités de façon éparse. Néanmoins, Jean Longère prépare l'édition des Sermones vulgares de Jacques de Vitry, et Simon Tugwell celle de la collection d'Humbert de Romans. Je travaille pour ma part sur la collection des Sermones ad status de Guibert de Tournai.

9. Le terminus post quem de la collection de Guibert, mort en 1284, est fourni par son prologue, qui mentionne que l'ouvrage est dédié à la mémoire du pape Alexandre IV, décédé en 1261. Humbert de Romans, mort en 1277, a composé le De eruditione predicatorum après 1266 (voir Alexander Murray, « Religion among the poor in thirteenth-century France : the testimony of Humbert de Romans », dans Traditio, t. 30, 1974, p. 258-324).

10. Sur la date de la collection, Jean Longère, Euvres oratoires de maîtres parisiens: étude historique et doctrinale, Paris, 1975, 2 vol. (Collection des Études augustiniennes, série Moyen Âge et Temps modernes, 4-5), t. II, note 227, p. 33.

11. E. Longpré notait déjà dans son introduction au Tractatus de pace..., p. xxIII, note 1 : «A Jacobo de Vitriaco multum dependet Gilbertus. »

12. Le premier à démontrer l'utilisation des Sermones vulgares par Guibert de Tournai fut Autbert Stroick, dans son article «Verfasser und Quellen der Collectio de scandalis Ecclesiae (Reformschrift des Fr. Gilbert von Tournay, ofM, zum II. Konzil von Lyon, 1274) », dans Archivum Franciscanum historicum, t. 23, 1930, p. 3-41, 273-199 et 433-466. Pour les sermons aux gens mariés, voir David L. d'Avray et M. Tausche, « Marriage sermons in ad status collections of the central Middle Ages », dans Archives d'histoire doctrinale et littéraire du Moyen Âge, 55e année, t. 47, 1980, p. 71-119. Pour les sermons de croisade, l'ouvrage de Christoph T. Maier, Crusade propaganda and ideology: model sermons for the preaching of the Cross, Cambridge-New York, 2000, comporte un appendice intitulé « The relationship between the crusade model sermons of Gilbert of Tournai and James of Vitry » (p. 250-263) qui démontre efficacement le jeu des emprunts et des innovations.

13. Nicole Bériou et François-Olivier Touati, «Voluntate Dei leprosus » : les lépreux entre conversion et exclusion aux XII et XIII ${ }^{e}$ siècles, Spolète, 1991 (Testi, studi, strumenti, 4), p. 44. 
modifié et reclassé la liste des status proposés par Jacques de Vitry ${ }^{14}$, mais il a surtout imprimé à la matière de sa source la forme du sermo modernus né au XIII ${ }^{\mathrm{e}}$ siècle ${ }^{15}$. Recomposant et réarrangeant ses emprunts, ne conservant qu'une sélection des exempla qui ont fait le succès de Jacques de Vitry, c'est indéniablement sa propre vision qu'il transmet, en poussant à son paroxysme l'art de la distinctio. Son style, fondé sur la puissance et l'efficacité de la structure plus que sur le souffle narratif, semble avoir trouvé un écho chez les lecteurs, puisque cette collection est de très loin celle dont le plus grand nombre de manuscrits nous sont parvenus ${ }^{16}$.

2. Les tables des manuscrits. - Le répertoire de Johannes Baptist Schneyer ${ }^{17}$ cite soixante manuscrits, dont quarante-neuf contiennent la collection complète, cinq simplement un ou plusieurs sermons isolés, et six sont à écarter ${ }^{18}$. Les éditions anciennes, lacunaires et très fautives, sont aussi à éliminer ${ }^{19}$. À ce jour, nous connaissons quatre-vingt-cinq manuscrits contenant l'ensemble de la collection ${ }^{20}$. Datant de la fin du xIII' ${ }^{\mathrm{e}}$ siècle au début $d u x v^{\mathrm{e}}$, ils sont conservés dans douze pays différents ${ }^{21}$. Cette tradition, encore mal connue ${ }^{22}$, semble néanmoins assez complexe.

14. Pour une liste des status distingués respectivement par Jacques de Vitry et par Guibert de Tournai, voir N. Bériou et F.-O. Touati, Voluntate Dei leprosus..., note 15, p. 39-40, et note 26 , p. 44-45.

15. Sur le sermo modernus et le nouvel art de prêcher, voir notamment : Nicole Bériou, L'avènement des maîtres de la parole: la prédication à Paris au XIII siècle, Paris, 1998 (Collection des Études augustiniennes, série Moyen Âge et Temps modernes, 31-32); ead., «Les sermons latins après 1200 », dans, The sermon, dir. Beverly M. Kienzle, Turnhout, 2000 (Typologie des sources du Moyen Âge occidental, 81-83), p. 363-448.

16. Si l'on s'en tient aux chiffres donnés dans le $R L S$, pour la collection de Guibert de Tournai 60 manuscrits sont signalés (t. II, p. 306-307), contre 15 pour celle de Jacques de Vitry (t. III, p. 220-221); le $R L S$ ne donne pas d'indication sur les manuscrits du De eruditione predicatorum d'Humbert de Romans, mais à titre de comparaison les références de 22 manuscrits sont données pour cette œuvre dans le répertoire de Thomas Kaeppeli, Scriptores ordinis Praedicatorum medii aevi, t. II, Rome, 1975, p. 287-288, et t. IV, Rome, 1993, p. 128).

17. $R L S$, t. II, p. 306-307.

18. Cracovie, Archiwum Kapituły Metropolitalnej, 174 (la cote donnée par le $R L S$ est ici erronée, il faut lire 170) ; Leipzig, Universitätsbibl., Rep. VI. 46.b ; Bibl. nat. Fr., lat. 15952 ; Bibl. mun. Troyes, mss. 1724 et 1748; Bibl. Vat., Pal. lat. 368, fol. 284-354v.

19. Louvain, 1473 et 1484; Paris, 1508 et 1513 ; Lyon, 1511.

20. Voir la liste, Annexe 1.

21. France (19 manuscrits), Italie et Vatican (13 et 4), Autriche (15), Allemagne (14), Pologne (6), Angleterre (6), Belgique (3), Espagne (2), Luxembourg (1), République Tchèque (1), Roumanie (1).

22. Ầ ce jour, l'aperçu le plus complet est celui donné par N. Bériou dans Voluntate Dei leprosus..., avec l'examen de 13 manuscrits pour l'édition de la série de sermons « ad leprosos et abjectos ». Aleksander Horowski prépare, outre son édition citée à la note 7 , une étude complète de la tradition du Rudimentum doctrine et des Sermones ad status, à paraître en 2009 dans la collection Subsidia scientifica franciscalia de l'Istituto storico dei Cappuccini. 
L'œuvre, qui figure sur la liste des manuscrits diffusés par le système de la pecia à Paris en 1304.23, comporte des variantes non seulement dans le texte, mais également dans l'intitulé des rubriques ainsi que dans le nombre et l'ordre des sermons ${ }^{24}$.

Pour la présente étude, il a été impossible de consulter l'ensemble des quatre-vingt-cinq témoins. La méthode appliquée a donc consisté, pour les manuscrits non disponibles directement ou en reproduction, à utiliser les descriptions fournies par les catalogues, en les complétant au besoin par des vérifications ponctuelles auprès des conservateurs ${ }^{25}$. Certains catalogues, en particulier les plus anciens, négligent en effet de signaler la présence d'index ou, lorsqu'ils le font, ne distinguent pas la simple table des matières et l'index analytique, ou encore indiquent un type erroné ${ }^{26}$. Au total, parmi les manuscrits de la collection, ceux qui contiennent un index analytique sont, dans l'état actuel de mes investigations, au nombre de

23. Heinrich Denifle et Émile Châtelain, Chartularium universitatis Parisiensis, Paris, 1889 , t. II, p. 107-112, $\mathrm{n}^{\circ}$ 642. Voir également La production du livre universitaire au Moyen Âge, exemplar et pecia : actes du symposium tenu au Collegio San Bonaventura de Grottaferrata en mai 1983, éd. Louis-Jacques Bataillon, Bertrand Guyot et Richard Rouse, Paris, 1988, réimpr. 1991.

24. Voir N. Bériou et F.-O. Touati, Voluntate Dei leprosus..., p. 84-91.

25. Je tiens à remercier, pour leur aide irremplaçable, les nombreux bibliothécaires qui ont facilité ce travail. Parmi bien d'autres, Enrica Coser (Bologne, Reale Collegio di Spagna) et Bernardo Pietri (Università di Bologna) m'ont notamment permis d'obtenir accès à une reproduction de très haute qualité des tables du ms. Bologne, Collegio di Spagna, 56. Klára Erdei (Kiel, Universitätsbibl.), Sonja Führer (Salzbourg, Bibl. der Erzabtei St. Peter), Suzanne Paul (Cambridge, Parker Library, Corpus Christi College), Heinz Ristory (Klosterneuburg, Stiftsbibl.), Eduard Scheiber (Brixen) et Friedrich Simader (Vienne, Österreichische Nationalbibl.) m'ont également apporté assistance et conseil pour l'obtention de reproductions de manuscrits de leurs fonds respectifs. À ces remerciements, il convient d'associer le personnel de l'Institut de recherche et d'histoire des textes, qui m'a donné accès aux microfiches de nombreux manuscrits évoqués ici. Carla Casagrande (Università di Pavia) et Aleksander Horowski (Istituto storico dei Cappuccini) ont eu la gentillesse de m'adresser une description des index de manuscrits que je n'avais pu consulter (Milan, Bibl. Ambrosiana, F. 57 sup. et P. 70 sup. ; Heiligenkreuz, Stiftsbibl., 144).

26. Ainsi, l'index analytique classique présent dans le ms. Luxembourg, bibl. de l'Athénée, 63, est mentionné dans le catalogue comme un « index des passages de l'Écriture sainte figurant dans le recueil précédent » (Nicolas van Werveke, Catalogue descriptif des manuscrits de la bibliothèque de Luxembourg, Luxembourg, 1894, p. 161). Cette confusion est due au système de numérotation des folios dans ce manuscrit, basé sur l'association de l'un des cinq premiers livres de la Bible et d'un chiffre romain. Les folios sont donc «numérotés » Gen. I à Lx, puis Exo. I à Lx, Levi. I à L, Numer. I à L, et enfin Deuter. I à LVIII, donnant l'impression au premier abord que les entrées de la table renvoient à des passages de l'Écriture. Ce système est pourtant décrit dans le prologue de la table, qui était connu de l'auteur du catalogue : « Gen., Exo., Levi., Numer., Deuter. : iste quinque dictiones non ponuntur hic pro quinque libris Moysi, sed accipiuntur materialiter, quia hec dictio Gen. continet sub numero suo LX folia, Exo. Lx, Levi. L, Numer. L, Deuter. LVIII. Et sic, per assignationem dictarum dictionum et dictorum numerorum, poteris quicquid quesieris in subsequentibus concordantiis valde velociter invenire » (fol. 282va). 
vingt-sept : un manuscrit contient trois index différents ${ }^{27}$, trois en contiennent deux ${ }^{28}$, le reste un seul ${ }^{29}$, soit un total de trente-deux index. Tous sont des index généraux ou tabule distinctionum (c'est-à-dire des termes faisant l'objet d'une distinctio dans un sermon), à l'exception de trois tables des exempla relevés dans le recueil de sermons, type d'index qui semble beaucoup plus rare ${ }^{30}$. Les renvois s'effectuent majoritairement au folio, plutôt qu'à un numéro de sermon dans la collection ${ }^{31}$.

Deux séries regroupent des tables clairement liées entre elles, présentant une liste de termes quasi identique. La première comprend quatre tables ${ }^{32}$, dont les entrées renvoient à un numéro de sermon associé à une lettre correspondant plus précisément à un paragraphe. Les trois tables ${ }^{33}$ de la seconde ne renvoient pas à un numéro de sermon, mais au folio; pour l'une des tables de ce groupe les renvois n'ont pas été complétés, tandis que le prologue de la table précise : Hec est tabula distinctionum totius libri secundum ordinem alphabeti. Numerus foliorum post qualibet dictionem debet $s c r i b i{ }^{34}$. Cela démontre que les renvois au folio, bien que dépendants du manuscrit, n'étaient pas incompatibles avec la transmission à l'identique de tables analytiques. L'ordre alphabétique, appliqué avec plus ou moins de rigueur, règne en maître pour le classement de ces tables, sans surprise pour des manuscrits de cette période. Cependant trois tables, réparties entre deux manuscrits conservés l'un à Paris et l'autre à Bologne ${ }^{35}$, présentent un usage particulier de l'ordre alphabétique, groupant les entrées en cinq ensembles correspondant aux voyelles, sans tenir aucun compte des consonnes pour le classement. Cet usage rare, et qui peut paraître incongru, appelle une étude plus détaillée.

27. Paris, Bibl. nat. Fr., lat. 16480.

28. Bologne, Collegio di Spagna, 56; Milan, Bibl. Ambrosiana, F. 57 sup. et P. 70 sup.

29. Pour la liste complète, voir Annexe 1 (manuscrits en italique).

30. Outre les tables des exempla présentes dans les manuscrits Bologne, Collegio di Spagna, 56, et Bibl. nat. Fr., lat. 16480, étudiées en détail dans cet article, le ms. Milan, Bibl. Ambrosiana, P. 70 sup. contient un index des exempla du recueil, sans lien avec les autres tables et beaucoup plus sommaire.

31. Neuf index seulement utilisent des renvois à un numéro de sermon : la tabula exemplorum de Bologne, Collegio di Spagna, 56, les trois tables de Bibl. nat. Fr., lat. 16480 (voir la description de ces deux manuscrits et de leurs tables plus bas), la table de Heiligenkreuz, Stiftsbibl., 144, et les quatre tables identiques dont les références se trouvent à la note suivante.

32. Graz, Universitätsbibl., 524; Salzbourg, Stiftsbibl. Sankt Peter, B XI 4 et B XII 38 ; Vienne, Österr. Nationalbibl., Palat. 5109.

33. Klosterneuburg, Stiftsbibl., 470 ; Vienne, Österr. Nationalbibl., Palat. 1297 et Palat. 1638.

34. Klosterneuburg, Stiftsbibl., 470, fol. 167.

35. Paris, Bibl. nat. Fr., lat. 16480 (désigné par P) et Bologne, Collegio di Spagna, 56 (désigné par $B$ ). 


\section{TRois TABLES SINGULIÈRES.}

1. Une autre logique de classement. - Ces tables sont de deux types : une tabula distinctionum, et deux tables des exempla du recueil. Elles regroupent leurs entrées en cinq ensembles, A, E, I, O et U, en fonction de la première voyelle apparaissant dans chaque entrée, quelle que soit sa position; soulignons qu'elles ne prennent pas en compte les $i$ et les $u$ à valeur consonantique ${ }^{36}$. Au sein de chaque ensemble, le classement suit simplement l'ordre d'apparition dans le recueil. Il est intéressant de noter que la référence donnée correspond ici au numéro du sermon, et non à un folio, laissant penser que cette table a été conçue pour être plus facile à copier et utiliser dans différents manuscrits de la collection.

La tabula distinctionum, transmise par le seul manuscrit parisien, est organisée en deux colonnes, l'une pour les entrées d'index, l'autre pour les renvois au numéro du sermon ${ }^{37}$. Les premières entrées de l'ensemble des termes correspondant à la voyelle A sont ainsi David, Sacerdos, Pascere, etc.; puis celles de l'ensemble des termes en E, Eligere, Stelle proprietates, Ferre, etc.

La table des exempla, transmise, elle, par les deux témoins sous une forme légèrement différente, présente une mise en page plus élaborée. Elle est organisée en trois colonnes : la première décrit le sujet de l'exemplum, la deuxième la leçon qui peut en être tirée, et la troisième renvoie au numéro du sermon. La première entrée de l'ensemble des exempla sous A est ainsi (d'après le ms. de Paris) : Exemplum de fratribus duobus - De merito servientium infirmis ; celle des E, Exemplum de leone - Contra gravantes pauperes subditos; celle des I, Exemplum de ligno arido - De obedientia, etc.

On notera au passage que cette table intègre la notion de «mots vides » : ainsi les entrées de beato Martino, de beato Francisco ou de beato Anthonio sont classées dans l'ensemble des exempla sous A et non sous $\mathrm{E}^{38}$; quodam de même est ignoré au bénéfice du premier mot significatif qui vient ensuite ${ }^{39}$. Il existe une certaine distance entre les deux tables : si elles ont un lien indéniable, la formulation des entrées dans $B$ s'éloigne fréquem-

36. Dans la tabula exemplorum de $P$ : «Ex. de vespertilione » est classé sous $\mathrm{E}$, « Ex. de viro quodam sancto » sous I, «Ex. de Johanne Elemosinario » sous O, «Ex. de juvene nobili », «Ex. de vulpe » et «Ex. de judice quodam » sous U (voir l'édition de la table, Annexe 3 A). On rencontre d'autres exemples dans la tabula distinctionum du même manuscrit : «Vasa » sous A, «Venire » et «Vestis » sous E, «Visitare », «Vita eterna », «Vidue », «Virginitas », «Virgo » et Virginitatis custodia » sous I, « Jocunditas » sous O, « Judex / Judicium » et « Judicare seipsum » sous U (édition de cette table, Annexe 2).

37. Voir les illustrations, Annexes 4 A et 4 B.

38. $P$, fol. 176 .

39. $B$ : «De quodam sapiente » est classé sous $\mathrm{A}$, « De quodam peregrino », « De quodam rege » sous $\mathrm{E}$, « De quodam impio » sous $\mathrm{I}$. 
ment mais insensiblement de celle de $P^{40}$. La deuxième colonne en particulier, celle qui donne la leçon à tirer de l'exemplum, subit régulièrement une reformulation légère tendant souvent vers un caractère plus générique $^{41}$.

2. Les manuscrits. - Un examen des deux manuscrits peut nous renseigner sur le processus de création de ces tables. En voici la description sommaire.

$P$. Paris, Bibliothèque nationale de France, lat. $16480: \mathrm{XIII}^{\mathrm{e}}-\mathrm{XIV}^{\mathrm{e}}$ siècle, parchemin, $185 \times 130$ mm, 176 fol., 2 colonnes à 41 lignes. Ancien manuscrit de la Sorbonne (anciennes cotes au fol. 2 : 751, 972). Au fol. 1, en marge de pied, l'instruction Fiant asseres et incatenetur in magna libraria Sorbone indique que le manuscrit a fait partie des livres enchaînés aux bancs de la grande bibliothèque de la Sorbonne ${ }^{42}$.

Contenu: fol. 1-v: index analytique, par ordre alphabétique «classique »; fol. 2-175v : Sermones ad status; fol. 175v-176 : index analytique, par ordre alphabétique des voyelles; fol. 176-v : index des exempla, par ordre alphabétique des voyelles (détérioré, de lecture très difficile par endroits).

Bibliographie : L. Delisle, Inventaire des mss. de la Sorbonne, Paris, 1870, p. 61 ; N. Bériou et F.-O. Touati, Voluntate Dei leprosus..., p. 88.

B. Bologne, Collegio di Spagna, 56: $\mathrm{xIV}^{\mathrm{e}}$ siècle, parchemin (et 2 fol. papier), $200 \times 245 \mathrm{~mm}$, III + 205 fol. , 2 colonnes à 41 lignes. Anciennes cotes : XL 5, E-VI-5. Ce manuscrit n'a pas été mentionné parmi les livres inclus dans la bibliothèque personnelle du cardinal Gil de Albornoz († 1367), fondateur du Collegio di Spagna à Bologne, et légués par lui au collège. Cependant, sur le plat antérieur, une note apparemment ignorée jusqu'ici permet d'affirmer que c'est bien là son origine. De la même main qu'une note similaire ${ }^{43}$ de Gil de Albornoz dans le ms. Bologne, Collegio di Spagna, 57 (dont on sait qu'il provient du legs), elle précise : Sermones quos emimus Parisius, archiepiscopus Toletanus, Nos Egidius ${ }^{44}$.

40. Dans un cas, cette reformulation a produit une absurdité dans le classement. Dans $B$, on trouve ainsi un exemplum «De famulis scolarium » classé sous E, ce que l'on ne peut comprendre qu'à la lumière de la table de $P$. Dans cette dernière, ce même exemplum est décrit comme « De servis Paris[ius] », et donc dûment classé sous E !

41. Cette reformulation fréquente m'a incitée à proposer l'édition séparée des deux tables, plutôt qu'une seule édition critique prenant en compte les deux témoins. Voir l'édition des tables des exempla, Annexes 3 A et 3 B.

42. Sur ces « catenati », voir Richard Rouse, « The early library of the Sorbonne », dans Scriptorium, t. 21, 1967, p. 42-71.

43. « Parisius dedit michi quidam frater predicator. Nos Egidius archiepiscopus Toletanus. » Voir Domenico Maffei, I codici del Collegio di Spagna di Bologna, Milan, 1992 (Orbis academicus, 5).

44. Je remercie Marc Smith pour son aide à la lecture particulièrement difficile d' « emimus ». 
Contenu : fol. 1-203, Sermones ad status; fol. 203v-204v, index analytique, par ordre alphabétique «classique»; fol. 204v-205 : index des exempla, par ordre alphabétique des voyelles.

Bibliographie: Celestino Piana, «Descriptio codicum Franciscalium necnon sancti Thomae Aquin[atis] in bibliotheca Albornotiana collegii Hispani Bononiae asservatorum », dans Antonianum, t. 17, 1942, p. 125; Marie-Hyacinthe Laurent, Fabio Vigilii et les bibliothèques de Bologne au début du XVI siècle, Cité du Vatican, 1943, p. 4, n. 19; Juan Gil Fernández, De codicibus Albornotianis ad Graecas Latinasque litteras pertinentibus commentarius [inventaire de 1511], Bologne, 1964, p. 18; A. García y García et C. Piana, «Los manuscritos filosófico-teológicos, históricos y científicos del Real Colegio de España de Bolonia », dans Salmanticensis, t. 14, 1967, p. 86 et 130-131; Domenico Maffei, I codici del Collegio di Spagna di Bologna, Milan, 1992 (Orbis academicus, 5), p. 76.

Un sondage réalisé sur le texte des trois sermons Ad leprosos et abjectos ${ }^{45}$ permet d'affirmer que $P$ et $B$ n'appartiennent pas à la même famille de manuscrits : $B$ ne présente aucune des lectures communes significatives qui permettent de rapprocher $P$ des manuscrits Paris, Bibl. nat. Fr., lat. 9606 et lat. 1494346 .

Chacun des deux manuscrits contient cent dix sermons, qui apparaissent dans le même ordre. En revanche, seul $P$ les numérote: les folios sont indiqués en chiffres arabes en haut à droite du recto, tandis que le numéro du sermon en cours figure au centre de la marge de tête, en chiffres romains. Mais cette numérotation comporte des erreurs, qui paraissent propres à ce manuscrit puisque essentiellement liées à un flottement occasionnel du scribe entre le décompte des sermons et celui des feuillets, si bien que la liste des sermons s'achève au numéro CXVIII au lieu de CX ${ }^{47}$.

45. $B$ : fol. $110-115 \mathrm{v} ; P$ : fol. $87 \mathrm{v}-92 \mathrm{v}$.

46. N. Bériou et F.-O. Touati, Voluntate dei leprosus..., p. 91, et édition des sermons Ad leprosos et abjectos, p. 129-155. Cette édition utilise $P$ comme l'un des témoins, mais non B.

47. Les erreurs de numérotation de $P$ portent sur les sermons suivants (par ordre d'apparition dans le manuscrit) : à partir du $10^{\mathrm{e}}$ sermon (numéroté $\mathrm{IX}$ ) et jusqu'au $71^{\mathrm{e}}$ sermon (numéroté LXX), retard de 1 par rapport à la numérotation logique (le scribe n'a sans doute pas vu la rubrique du $10^{\mathrm{e}}$ sermon, pas très visible dans ce manuscrit). Au $72^{\mathrm{e}}$ sermon, numéroté LXXII, un saut de 1 permet de retomber sur le bon numéro : il semble qu'à cet endroit le scribe, étourdi, ait commencé à numéroter séquentiellement les folios, et non plus les sermons, à partir de 70 ; le folio précédent était en effet numéroté LXXI (alors qu'il correspondait toujours au $70^{\mathrm{e}}$ sermon), mais le I a été effacé. Le $73^{\mathrm{e}}$ sermon n'est pas numéroté (de façon logique, car il a été copié en continu avec le sermon précédent, séparé par un simple pied-de-mouche), mais malgré cet oubli la série garde une numérotation correcte de 74 à 88. Les erreurs suivantes semblent encore liées aux étourderies d'un scribe hésitant entre la logique de numérotation séquentielle des sermons et des feuillets, puisque les décalages constatés sont cohérents avec le nombre de feuillets qui séparent le numéro erratique du précédent numéro de sermon. Ainsi, un saut de 1 décale à nouveau la numérotation à partir du $89^{\mathrm{e}}$ sermon (numéroté $\mathrm{LXXXX}, 2$ fol. après le sermon précédent numéroté 
Les trois tables de $P$ renvoient au numéro du sermon, en utilisant la numérotation erratique présente dans le manuscrit, et sans jamais faire référence aux folios : on peut donc penser qu'elles ont été confectionnées sur $P$ ou tout au moins adaptées à celui-ci pour que le système de références corresponde. $B$, en revanche, est folioté mais les sermons n'y sont pas numérotés. Cependant, si la table des distinctions selon l'ordre alphabétique classique de $B$ renvoie bien aux folios, la table des exempla renvoie aux mêmes numéros de sermon que celle de $P$ (c'est-à-dire en suivant la numérotation erratique propre au manuscrit parisien). Il paraît donc clair que la tabula exemplorum de $B$ dépend de celle de $P$. Il faut noter également que, si le scribe de cette table ne s'est pas inquiété des erreurs de numérotation ni de la difficulté d'utiliser un tel système de référence avec un manuscrit où les sermons ne sont pas numérotés, l'incohérence objective qui résultait de l'importation fidèle de ce système dans $B$ ne lui a pas totalement échappé. Il devait savoir que la collection ne contenait que cent dix sermons au total, et a donc été gêné par la présence, dans la table qu'il copiait, d'entrées renvoyant à des sermons portant un numéro plus élevé. Mais au lieu de vérifier la table (dont les renvois sont donc faux à partir de la première erreur de $P$ ) et de tenter de corriger la numérotation d'après $B$, il a résolu ce problème en éliminant les entrées dont le numéro de renvoi dépasse le nombre réel de sermons dans la collection 48 ! Seule a réchappé une entrée numérotée « 116 », car elle avait d'abord été notée par erreur «106 », et visiblement lors de la correction le scribe n'a plus remarqué ce numéro incohérent ${ }^{49}$.

La méthode de composition des tables analytiques semble reposer, à la manière habituelle, sur un premier repérage et signalement des éléments importants dans les marges du manuscrit, qu'un feuilletage systématique a permis ensuite de compiler sous forme d'index en relevant le numéro du feuillet ou du sermon.

LXXXVIII) et jusqu'au 95 e sermon. Viennent alors s'ajouter de nouveaux sauts : plus 1 à partir du $96^{\mathrm{e}}$ sermon (LXXXXVIII, 2 fol. après le sermon précédent numéroté LXXXXVI), plus 1 encore à partir du $99^{\mathrm{e}}$ sermon (CII, 2 fol. après le sermon précédent numéroté $\mathrm{C}$ ), plus 1 à partir du $101^{\mathrm{e}}(\mathrm{CV}, 2$ fol. après le sermon précédent numéroté $\mathrm{CIII})$. Le saut de 2 à partir du $102^{\mathrm{e}}$ sermon (CVIII, 1 fol. après le sermon précédent numéroté CV) doit avoir une autre origine, mais le dernier saut de 2 au $105^{\mathrm{e}}$ sermon conforte lui aussi l'hypothèse d'erreurs dues à un va-et-vient entre le décompte des sermons et celui des folios (CXIII, 3 fol. après le sermon précédent numéroté $\mathrm{CX}$ ).

48. Dans les E, l'exemplum «de Hechyne » (renvoi au sermon 118) ne figure pas dans la table de $B$; dans les $\mathrm{O}$, le scribe a copié l'entrée correspondant à l'exemplum « de Moyse » (s. 115), a commencé à copier l'entrée de l'exemplum « de moniali quodam » (s. 116) mais s'est arrêté en cours de route, et a barré ces deux lignes. Il n'a pas indiqué non plus l'entrée qui suit dans $P$, bien que celle-ci, sans doute placée en fin de liste à la suite d'un oubli, n'entre pas dans les références dépassant le nombre réel de sermons dans la collection (exemplum «de quodam inaniter gloriante», s. 35). Dans les U, l'exemplum «de judice quodam » (s. 115) ne figure pas non plus.

49. $B$, fol. $204 \mathrm{v}$. 
Dans $B$, les tables sont de deux mains différentes, et seuls les éléments qui figurent dans la tabula distinctionum selon l'ordre alphabétique classique ont été repérés en marge du manuscrit. Les exempla du recueil n'ont pas été repérés par leur description en marge, tout au plus une mention exemplum, ce qui vient confirmer que la tabula exemplorum est bien une pièce rapportée, copiée dans $B$ d'après un autre manuscrit.

Dans $P$, les trois tables sont chacune d'une main différente. L'écriture de la tabula distinctionum classée en fonction de la première voyelle présente des similitudes avec celle des entrées d'index portées en marge, face aux distinctions, avec une sorte de pied-de-mouche devant le mot-clé, ainsi qu'avec la numérotation des sermons dans le corps du manuscrit, bien que la brièveté de ces mentions limite les éléments de comparaison. Il est donc possible que le même scribe en soit responsable. La table des distinctions par ordre alphabétique classique, en tête du volume, présente d'ailleurs une erreur : Federe ${ }^{50}$ au lieu de Sedere, résultant sans doute d'une mauvaise lecture de l'entrée d'index portée en marge ${ }^{51}$. Si le scribe était le même, on peut supposer qu'il aurait difficilement commis cette erreur. Il faut souligner que ces deux tabule distinctionum de $P$ ne semblent pas être un remaniement l'une de l'autre, mais le fruit de deux relevés distincts, et plus ou moins exacts, des mêmes entrées marginales. Dix-huit entrées sur cent soixante-sept figurent uniquement dans la première table ${ }^{52}$, vingt-deux sur cent soixante-huit uniquement dans la seconde ${ }^{53}$ : on peut attribuer ces différences à des erreurs indépendantes survenues au moment où chacune des tables a été dressée dans $P$, à partir du relevé des entrées marginales. Si l'on observe ces dernières, dans le corps du manuscrit, on constate que les entrées retenues seulement dans l'une ou l'autre des deux tables sont par ailleurs de la même main, ce qui exclut l'hypothèse de la composition concurrente de ces tables.

La compilation de la tabula exemplorum de $P$ pose plus de questions. En marge du manuscrit, une première main a repéré les exempla qui la composent avec la simple mention exemplum, puis une autre main a parfois ajouté une brève description, plus courte et légèrement différente de ce que l'on trouve dans la table. Ainsi, alors que la première colonne de la table, celle

50. $P$, fol. 1 .

51. $P$, fol. 38 .

52. «Crux 》 ( 2 occurrences, avec renvoi au sermon 57 et 59 respectivement), « Desiderium » (s. 60), «Devotio » (s. 77), « Jerusalem » (s. 118), « Letitia » (3 occurrences, s. 116, 117 et 118), « Mendicus » (s. 56), « Merces » (s. 69), « Negociari » (2 occurrences, s. 64 et 65), « Peregrinus » (s. 60), « Regnum » (s. 83), « Servi » (s. 91), « Thesaurus » (2 occurrences, s. 81 et 82), « Venire » (s. 64).

53. «Civitas 》 (3 occurrences, s. 33, 34, 99), « Civitas Maria » (s. 32), « Civitas paradisi » (s. 35), «Dirigere » (s. 26), «Discere » (s. 29), «Fictio » (s. 29), «Filii » (s. 7), « Filius » (s. 8), « Ignis » (2 occurrences, s. 16 et 17), « Lignum » (s. 19), «Lingua » (s. 17), « Minister 》 (2 occurrences, s. 9 et 20), « Ministerium » (s. 4), «Misericordia » (s. 47), «Rivuli » (s. 17), « Scriptura » (s. 28), « Spiritus sanctus » (s. 17), « Timor » (s. 7). 
sur laquelle porte le classement, comporte le sujet de l'exemplum, la main qui a porté les descriptions en marge semble plus attentive à la leçon qui peut être tirée, reprise dans la table en deuxième colonne ${ }^{54}$. Cet index analytique ne résulte donc pas d'une compilation directe des descriptions marginales. On peut penser que la composition très structurée de cette table, en trois colonnes thématiques, a réclamé une étape intermédiaire de mise en forme. Deux hypothèses peuvent être envisagées. Soit la table a d'abord été préparée sur un brouillon qui ne nous est pas parvenu, puis recopiée dans $P$; le faible espace disponible dans ce manuscrit expliquerait alors la mise en page assez peu soignée, qui contraste avec l'analyse détaillée des exempla. Soit la table de $P$ dérive d'un modèle déjà mis en forme dans un autre manuscrit de la collection de Guibert, que le copiste aurait emprunté tout en adaptant la numérotation des renvois au contenu de son manuscrit. En tout cas, la présence dans la tabula exemplorum de $B$ de renvois identiques à ceux de $P$, avec les erreurs de numérotation propres à ce manuscrit $^{55}$, oriente vers une dépendance de la table de $B$ envers celle de $P$.

3. Pistes d'interprétation. - Les tables analytiques alphabétiques sont, à partir du XIII ${ }^{\mathrm{e}}$ siècle, des éléments courants dans les manuscrits, y compris les sermonnaires. En revanche, si les recueils d'exempla pouvaient comporter des index ${ }^{56}$, les tables des exempla d'un recueil de sermons semblent extrêmement rares. Il est donc étonnant de les voir ici composées selon un principe alphabétique lui aussi fort peu courant, et dont la logique paraît aujourd'hui déroutante.

La littérature ne semble pas donner d'autres exemples d'index appliquant le même classement que les tables de $P$ et $B$; mais quelques autres outils de travail médiévaux témoignent d'une même priorité donnée aux voyelles. Les Glossae affatim, glossaire du haut Moyen Âge, appliquent un principe alphabétique analogue à celui qui est évoqué ici, mais sans totalement éliminer les consonnes : les entrées sont classées en fonction de leur première lettre, puis de la première voyelle après cette lettre, quelles que soient les autres lettres éventuellement présentes dans l'intervalle ${ }^{57}$. Mary

54. Pour l'exemplum décrit dans la table de $P$ comme « Ex. de ligno arido - De obedientia », la mention marginale est « Exemplum de obedientia » (fol. 65); pour « Ex. de advocato infideli - De protractione omne », «Exemplum de avaritia » (fol. 76v); pour « Ex. de Scipione - De castitate », «Exemplum de castitate 》 (fol. 77) ; pour « Ex. de comite quodam Campanie - De operibus pietatis», «Exemplum de operibus misericordie et pietatis» (fol. 82); pour « Ex. de angelo - De fetore peccantium », « Exemplum de superbia et fetore luxurie seu fetore peccantium » (fol. 91v), etc.

55. Voir ci-dessus, note 47.

56. Voir Jean-Claude Schmitt, «Recueils franciscains d'exempla et perfectionnement des techniques intellectuelles du $\mathrm{XIII}^{\mathrm{e}}$ au $\mathrm{xv}^{\mathrm{e}}$ siècle, » dans Bibliothèque de l'École des chartes, t. 135,1977, p. 5-22.

57. Lloyd W. Daly, Contributions to a history of alphabetization in Antiquity and the Middle Ages, Bruxelles, 1967 (collection Latomus, 90), en particulier p. 70 : « It results, for 
Carruthers signale par ailleurs l'existence d'un index composé selon un principe voisin dans un manuscrit du XIII ${ }^{\mathrm{e}}$ siècle du commentaire des Sentences par Richard Fishacre ${ }^{58}$ : comme dans les tables étudiées ici, le classement ne dépend pas de l'initiale du mot, mais de ses voyelles. Cependant, alors que dans nos manuscrits seule la première voyelle est prise en compte et que le numéro du sermon fournit la seconde clé de classement au sein de chaque ensemble, l'index de l'œuvre de Richard Fishacre considère plus systématiquement les syllabes et leurs combinaisons de voyelles, classant d'abord les monosyllabes, puis les polysyllabes en fonction des voyelles apparaissant dans chaque syllabe. Cet index se conclut d'ailleurs par un paragraphe précisant que le classement repose sur «l'ordre des voyelles dans l'alphabet» et expliquant comment l'utiliser «pour trouver rapidement les choses qui sont contenues dans le livre qui précède » ${ }^{59}$, tout comme la tabula exemplorum de $P$ se veut utilis et facilis ad inveniendum omnia exempla ibidem posita et contenta ${ }^{60}$. Loin d'être le produit oiseux de quelques facétieux scholares préfigurant l'Oulipo ${ }^{61}$, la logique de l'ordre alphabétique selon les seules voyelles, telle que la présentent les tables de $P$ et $B$, participait bien de la quête du statim invenire.

Deux pistes peuvent à mon sens permettre d'expliquer l'adoption de cette logique : la recherche à travers les voyelles d'éléments stables dans la graphie des entrées, et une attention prêtée à la mnémotechnique.

Pour l'index du commentaire des Sentences de Fishacre, Mary Carruthers explique le mode de classement par les méthodes d'apprentissage du latin et de mémorisation des mots alors en cours, fondées sur les syllabes (consonne et voyelle associées), tout en s'interrogeant sur le « mystère » des consonnes négligées. C'est peut-être Papias qui nous donne une clef de compréhension : dans la préface de son dictionnaire, là où il expose les règles de classement de l'ouvrage (un ordre alphabétique classique), il précise que cet ordre peut parfois varier «à cause de la graphie de certaines lettres. Par exemple, certains écrivent hyena avec un $i$, d'autres avec un $y$, ou par aspiration [c'est-à-dire sans $h$ ], et la plante que certains appellent verbena, d'autres l'appellent berbena $\gg{ }^{62}$. Dans une culture écrite soumise à

example, that words beginning in $f l a$ - and fra-may precede words beginning in $f a$-, and that 144. glosses intervene between affatim and affecta ».

58. Mary Carruthers, The book of memory : a study of memory in medieval culture, $2^{\mathrm{e}}$ éd., Cambridge (Mass.), 2008, p. 150-151 (ms. Oxford, New College, 112).

59. Ibid., p. 150.

60. $P$, fol. 176 .

61. Ouvroir de littérature potentielle, groupe de littérature expérimentale fondé à Paris en 1960 .

62. Texte original : «Cum vero earundem litterarum primus, secundus, tertius ordo observatus fuerit, interdum ratio variabitur. Verbi gratia, hyena a quibusdam per .i., ab aliis per.$y$. vel per aspirationem scribitur. Et quam verbenam quidam, alii berbenam nominant herbam » (ma traduction); prologue édité dans Lloyd W. Daly et Bernardine A. Daly, «Some techniques in mediaeval Latin lexicography », dans Speculum : a journal of mediaeval studies, t. 39/2, 1964, p. 229-239, citation p. 231. 
des règles orthographiques peu fixes, les voyelles des premières syllabes d'un mot ont donc pu être perçues comme des éléments finalement plus stables, plus significatifs. Il faut reconnaître en effet que ce système ne laisse pas subsister d'ambiguïté sur l'emplacement des mots comportant un $h$ initial muet parfois omis dans la graphie, de mots commençant indifféremment par $p h$ ou $f$, ni d'un mot comme Christus, que l'on retrouve dans les tables alphabétiques classiques soit sous l'entrée C, soit sous $\mathrm{X}$, selon sa graphie abrégée xps. Un glissement dans ce sens semble d'ailleurs s'être effectué dans certaines tables ordonnées globalement selon l'ordre alphabétique classique, mais avec des exceptions pour les entrées commençant par $h$ : celles-ci ont pu être classées selon la lettre suivante, tout en conservant leur graphie classique; dans un manuscrit du xIV $^{\mathrm{e}}$ siècle conservé à la Bibliothèque Vaticane, les entrées homo et honorare se trouvent ainsi classées sous $\mathrm{O}^{63}$.

Enfin, on peut également envisager qu'un souci de mémorisation plus aisée ait poussé les scribes à adopter cette répartition des entrées en cinq ensembles correspondant à chacune des voyelles. La suite «a, e, i, o, u» était suffisamment présente dans les esprits pour que Robert de Basevorn l'ait utilisée comme support mnémotechnique de la division du thème d'un sermon sur la Passion ${ }^{64}$. Cette division des entrées d'une table en cinq ensembles, au lieu d'une vingtaine en suivant l'ordre alphabétique complet, est d'ailleurs conforme au topos, repris à son compte par Guibert de Tournai lui-même dans le Rudimentum doctrine ${ }^{65}$, qui voulait qu'une division en peu de membres soit préférable car plus facile à mémoriser.

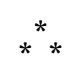

Avec vingt-sept manuscrits sur quatre-vingt-cinq dotés d'au moins une table d'index, les Sermones ad status de Guibert de Tournai offrent un bel exemple du soin qu'ont mis les lecteurs médiévaux à équiper d'instruments de recherche cette importante et imposante collection. Si l'ordre alphabétique, système de référence utilisé par tous les témoins, ne surprend guère dans ces manuscrits produits à partir de la fin du XIII ${ }^{\mathrm{e}}$ siècle, la logique centrée sur les voyelles, mise en œuvre pour le classement des trois tables étudiées ici plus en détail, est bien éloignée de nos usages, et paraît aujourd'hui difficile à appréhender. Pourtant, bien que peu répandu, cet aménagement apporté à l'ordre alphabétique atteste non seulement un

63. L. Pellegrini, Indici per predicare..., p. 131 (Bibl. Vat., lat. 4368).

64. M. Carruthers, The book of memory..., p. 132.

65. «Caveas autem in divisionibus ne sint membra plurima, nam divisio quanto fit per membra pauciora tanto melior, quanto plura deterior », et «Fit enim parcium divisio ut cesset confusio et adjuvetur memoria, nam facilius retinentur et memorie commendantur et ex diffinitionibus elicitur » (Paris, Bibl. nat. Fr., ms. lat. 15451, fol. 225a et 225b respectivement). 
rapport plus vocalique que le nôtre à l'écriture, mais aussi l'inventivité des techniques mises en œuvre pour faciliter l'accès au travail intellectuel et sa mémorisation.

\section{Marjorie Burghart.}

\section{ANNEXES}

1

Liste des manuscrits de la collection complète des «Sermones ad status»

(À l'exclusion des manuscrits contenant un ou plusieurs sermons isolés)

Les manuscrits ne figurant pas dans la liste du $R L S$ sont précédés d'un astérisque ; sont donnés en italique les manuscrits qui comportent au moins un index analytique.

*Alba Iulia, Bibl. Batthyanyana, 211.

Angers, Bibl. mun., 250.

Anvers, musée Plantin, 102 (cat. : 106).

*Arras, Bibl. mun., 325 (202).

Assisi, Bibl. del Sacro Convento, 486, 501.

*Augsburg, Universitätsbibl., Cod. II. 1. $2^{\circ}$ 67.

Bologne, Collegio di Spagna, 56.

Bordeaux, Bibl. mun., 293.

Braunschweig, Stadtbibl., 50.

${ }^{*}$ Brixen, Klarissenkloster, G. 43 (MS 33).

Bruges, Bibl. mun., 289.

Bruxelles, Bibl. royale, 1890 (4284).

Burgo de Osma, Bibl. capitular, 146.

${ }^{*}$ Cambridge, Peterhouse, 200.

${ }^{*}$ Cambridge, Corpus Christi, 506.

${ }^{*}$ Châlons-en-Champagne, Bibl. mun., 13.

Douai, Bibl. mun., 505.

Firenze, Bibl. naz. centrale, C. IX. 1084, J.X. 50.

${ }^{*}$ Gießen, Universitätsbibl., Hs 824.

Graz, Universitätsbibl., 233, *524.

${ }^{*}$ Hamburg, Staats- und Universitätsbibl., Petri 31.

Heiligenkreuz, Stiftsbibl., 144, 311.

Kiel, Universitätsbibl., 19.
Klosterneuburg, Stiftsbibl., 470.

*Kraków, Bibl. Jagiellonska, 668, *691, *1221, *1493.

*Kraków, Archiwum Kapituły Metropolitalnej, 170.

*Leipzig, Universitätsbibl., 623.

*Lilienfeld, Stiftsbibl., 48, *73.

*London, British Library, Royal 10 A. XI.

Luxembourg, Bibl. de l'Athénée, 63.

Marseille, Bibl. mun. 392 (Eb 157).

${ }^{*}$ Mainz, Stadtbibl., Hs. I 401.

*Melk, Stiftsbibl., 764.

*Milano, Bibl. Ambrosiana, F 57 sup., ${ }^{*}$ G. 34 inf., P. 70 sup.

München, Bayerische Staatsbibl., Clm 14570, Clm 18709, Clm 19603, Clm 21629.

Padova, Bibl. Antoniana, 469.

Paris, Bibl. nat. Fr., lat. 9606, lat. 14943, lat. 15941, lat. 15943, lat. 15953, lat. 16479, lat. 16480.

*Poitiers, Bibl. mun., 100.

Praha, Knihovna Metropolitní Kapituli, 960 (F.C/2).

Reun, Stiftsbibl., 30.

*Roma, Bibl. Casanatense, 605. 
Saint-Omer, Bibl. mun., 315.

*Salzburg, Stiftsbibl. Sankt Peter, B XI 4, BXII 38.

*Sankt Paul im Lavanttal, Benediktinerstift St. Paul, Cod. 98/4.

*Soissons, Bibl. mun., 135.

Subiaco, Santa Scolastica, (CCXXIII) 226.

*Tours, Bibl. mun., 489.

Trier, Stadtbibl., 338/2010.

Troyes, Bibl. mun., 1143, 1504.

Valencia, Bibl. de la catedral, 294.
*Vaticano (Città del), Bibl. Apostolica Vaticana, Borg. lat. 107, 217, 241, *Ottob. lat. 861.

Venezia, Bibl. Marciana, Lat. II. 5.

*Wien, Österreichische Nationalbibl., Palat. 1297, *Palat. 1638, *Palat. 1658, *Palat. 5109.

Wolfenbüttel, Herzog August Bibl., Aug. 3663.

Worcester, Cathedral, F. 36, F. 77, Q. 57.

*Wrocław, Bibl. Uniwersytecka, I F 657.

\section{2}

Édition de la «tabula distinctionum 》

du ms. Paris, Bibl. nat. Fr., lat. 16480, fol. 175v-176.

[fol. 175v]

A

David

Sacerdos .........................

Pascere ................

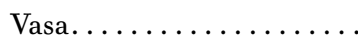

Apropinquare ...........

Sanctificatio..............

Tabernaculum ...........

Sanctificare .............

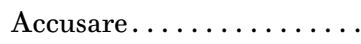

Pax ..................

Audire................

Sanctus................

Ascendere..............

Karitas ...............

Habitare ..............

Tacere, Taciturnitas ......

Aperire Dei ............

Sapiens, Sapientia........

Pascere ...............

Ager ..................

Ambulare .............

Karitas ...............

Radix

Frater.................

Habitare ..............

Sapiens.

66. Add in marg.
Ambulare

s. XLVII

Pater

s. XLVIII

Sanare....................

s. LI

Salvare ...............

ibidem

Gaudium ..............

s. V

Habundare.............

Abstinere a malo .........

ibidem

Gladius................

s. LX

s. LXI

Labor .................

Labor .................

s. LXVI

s. LXVII

ibidem

s. XIII

s. XIIII 66

Abscondere ............

s. LXXXIII

Aqua ................ s. C

s. XV

s. XVIII

s. XIX

Panis..................

s. CIIII

Matrimonium .......... s. CXIII

Mandata.............. s. CXIIII

ibidem

s. XX

s. XXIII

E

s. XXVIII

s. XXXI

s. XXXIX

s. XL

s. XLII

s. XLIII

ibidem

s. XLIIII

ibidem

s. XLVI
Eligere ...............

Stelle proprietates.......

Ferre................

Venire ................

Mensa.................

Electio ...............

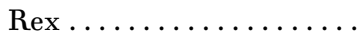

Ferre................

Reclinare .............

Vestis.................

Sedere................

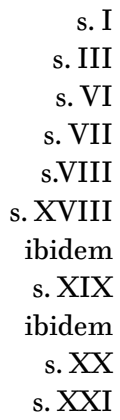

s. VII s.VIII XVIII ibidem s. XIX bidem s. XX s. XXI 
Levare.

s. XXIIII

Dilectio

s. LXXII

Levare oculos...........

s. XXV

s. XXVII

Sensus................

Seminare

Crescere

Relinquere

Sequi. .

Temptatio. .

Temptare

\section{I}

Ministerium...........

Filii .................

Timor................

Filius................

Minister . . . . . . . . . . . .

Visitare ..............

Ignis ................

Spiritus sanctus..........

Rivuli ...............

Lingua ................

Ignis .................

Lignum................

Minister...............

Dirigere ...............

Scriptura .............

Fictio................

Discere ...............

Civitas Maria...........

Civitas................

Civitas................

Civitas paradisi ..........

Israel. ................

Vita eterna .............

Ministerium...........

Fidelis...............

Misericordia ...........

Misericordia ...........

Misericordia ...........

Tribulatio.............

Tribulatio.............

Invenire .............

Signum crucis ..........

Signum diaboli ..........

Signum crucis ..........

Filii .................

Divitie................

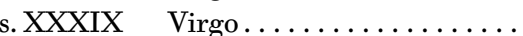

s. XL Virginitatis custodia ......

XLI

ibidem

s. LIIII

s. LV

Civitas.

Minister

[fol. 176] O

Providentia Dei

Solis proprietates ........

s. IIII

Oliva ...

s. VII

ibidem

s. VIII

s. IX

s. XIIII

s. XVI

s XVII

ibidem

ibidem

ibidem

s. XIX

s. XX

s. XXVI

s. XXVIII

s. XXIX

ibidem

s. XXXII

s. XXXIII

s. XXXIIII

s. XXXV

s. XXXVI

s. XLII

s. XLV

ibidem

s. XLVII

s. XLVIII

s. XLIX

s. L

s. LII

s. LV

s. LVII

s. LVIII

s. LIX

s. LXII

s. LXIII
Domus

Nova ..

Montes................

Oculus................

Columpna..............

Domus ................

Holocaustum ...........

Solitudo, Solus. . . . . . . . . .

Oculos levare ............

Docere Dei.............

Communicare ...........

Honestas. . . . . . . . . . . .

Gloria ................

Gloria ................

Gloria .................

Gloria ................

Propheta................

Honestas. . . . . . . . . . . .

Ordo ..................

Corvus ................

Consideratio ...........

Novitas . . . . . . . . . . . .

Jocunditas . . ..........

Bonum ...............

Mors .................

Portare ...............

Obsecrare...............

Opera vel Operari ........

Operari................

Honor . . . . . . . . . . . . .

Domus ...............

Ornamentum...........

Ornatus ..............

Mors
Congregare s. LXXIIII

s. LXXV

s. LXXVI

s. LXXVII

s. LXXXXIX

s. CV

s. II

s. III

s. VIII

s. XI

s. XII

s. XV

s. XVI

ibidem

s. XIX

s. XX

ibidem

s. XXII

s. XXV

s. XXVI

s. XXX

ibidem

s. XXXII

s. XXXIII

s. XXXIIII

s. XXXV

s. XXXVI

s. XXXVII

s. XXXVIII

s. XXXIX

s. XL

s. XLII

s. XLIIII

ibidem

s. LVI

ibidem

s. LX

s. LXVIII

s. LXIX

s. LXX

s. LXXII

s. LXXVIII

s. LXXIX

s. LXXXV 


\begin{tabular}{|c|c|c|c|}
\hline Mors . & s. LXXXVI & Filii & s. XV \\
\hline Mortuus . & s. LXXXVIII & Vulpes. & s. XXXVI \\
\hline Obedire. . & s. LXXXXII & Fundare & s. XLIII \\
\hline Domus ... & s. LXXXXIII & Unitas.......... & s. XLIIII \\
\hline Doctrina.... & s. LXXXXIIII & Judex, Judicium. . . . . . . . . & s. XLVI \\
\hline Docere & s. LXXXXV & Judicare seipsum . . & s. XLVII 67 \\
\hline Oratio. . & s. LXXXXVIII & Munus & s. LXI \\
\hline Confirmatio.. & s. CII & Nuptie.... & s. LXXX \\
\hline Confessio . & s. CVIII & Pulcritudo & s. LXXXIIII \\
\hline Oleu & s. CX & Puer & s. LXXXXVI \\
\hline Conventus ... & s. CXVIII & Ungere $\ldots \ldots \ldots \ldots \ldots$ & CIX \\
\hline & & Ungere $\ldots \ldots \ldots \ldots \ldots$ & CX \\
\hline U & & \multirow{4}{*}{\multicolumn{2}{|c|}{$\begin{array}{l}\text { Explicit tabula super summam Guiberti } \\
\text { ad omnem statum, utilis et facilis } \\
\text { ad inveniendum omnes distinctiones }\end{array}$}} \\
\hline Superioritas..... & s. II & & \\
\hline Lune proprietates ........ & s. III & & \\
\hline Mundicia $\ldots \ldots \ldots \ldots \ldots$ & s. VI & & \\
\hline
\end{tabular}

$3 \mathbf{A}$

Édition de la «tabula exemplorum » du ms. Paris, Bibl. nat. Fr., lat. 16480, fol. 176-v.

[fol. 176] Incipit tabula super summam istam, utilis et facilis ad inveniendum omnia exempla ibidem posita et contenta.

A

Ex. de fratribus duobus ....... De merito servientium infirmis ..... s. XXXVIII ${ }^{68}$

Ex de advocato infideli ........ De protractione [omne] .......... s. XLVI

Ex. de beato Martino ......... De operibus misericordie .......... s. XLIX

Ex. de beato Francisco ........ De operibus misericordie .......... s. XLIX

Ex. de beato Anthonio ....... De operibus misericordie .......... s. XLIX

Ex. de angelo ............. De fetore peccantium ............ s. LVI

Ex. de beato Martino ........ De cruce .................. s. LIX

Ex. de Karolo Magno .......... Contra nolentes audire sermonem..... s. LXI

Ex. de Alexandro........... Contra adulatores ............. s. LXI

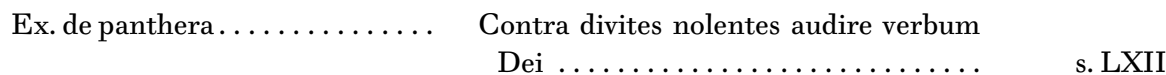

Ex. de avaro ............ Contra eos qui custodiunt sua [vesti-

menta $]$..................... s. LXVI

Ex. de Paulo abbate.......... Contra otiosos nolentes laborare ..... s. LXVIII

Ex. de marito et uxore ........ Contra conjuges non honorantes se.... s. LXX

Ex. de matre silvestri caprioli ... De obedientia ad parentes.......... s. LXXXXV

Ex. de sapiente quodam ........ De consideratione mortis .......... s. LXXXXV

67. XLVII] Il est possible qu'ily ait eu initialement un troisième I gratté ensuite. L'appel de cette entrée se trouve effectivement au sermon 47 et non 48 , la première table en tête du ms. comporte une erreur.

68. Suit une ligne effacée « Ex. de do[...]- De [...]» 
E

Ex. de leone .................

Ex. de peregrino quodam $\ldots .$.

Ex. de rege quodam ...........

Ex. de serpente

Ex. de vespertilione. . . . . . . . . .

Ex. de rege Philippo ..........

[fol. $176 \mathrm{v}$ ] E

Ex. de leone ................

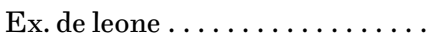

Ex. de heremita ............

Ex. de rege habente III filias ...

Ex. de Herode ...............

Ex. de demone..............

Ex. de Thecla...............

Ex. de servis Paris[ius $]. . . \ldots \ldots$

Ex. de servo quodam..........

Ex. de beato Bernardo ..........

Ex. de Renardo ..............

Ex. de feneratore quodam ......

Ex. de Hechyne .............

\section{I}

Ex. de ligno arido . ...........

Ex. de Scipione .............

Ex. de milite quodam ..........

Ex. de imperatore Troiano [sic]. .

Ex. de milite quodam ..........

Ex. de philosopho Crate........

Ex. de milite. .............

Ex. de impio quodam ..........

Ex. de illo furto ..............

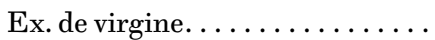

Ex. de milite quodam ..........

Ex. de divite quodam .........

Ex. de viro quodam sancto......

Ex. de filio quodam malo .......

Ex. de ciconia ...............

Ex. de milite quodam
Contra gravantes pauperes subditos ... s. XL

De gaudio contempla[tionis] .........

De judicantibus sine personarum acceptione ....................

Contra adulatores ................

Contra gravantes pauperes..........

De nocentibus sibi ad invicem ........

Contra nocentes vicinis.

s. LXI

Quod nullum bonum irremuneratum ..

Contra otiosos ...................

De honore parentum.............

De amentibus propter uxores.........

Contra fatuas mulieres ............

De castitate mulierum.............

De furto infidelium servorum. ........

contra pigros servos...............

De contemptu mundi. .............s

Contra cito redeuntes ad peccata......

Contra usurarios ...............

De beneficiis, videlicet quanto plus acceptantur pauca de proprio quam plura de alieno..................

s. LXI

s. LXI

s. LXII

s. LXII

s. LXVI

s. LXVI

s. LXVIII

s. LXX

s. LXXII

s. LXXII

s. LXXVI

s. LXXXXI

s. LXXXXI

LXXXXIIII

s. LXXXXV

s. CXVI

s. CXVIII

De obedientia.................

De castitate...................

s. XXXVIII

s. XLVI

De cruce et contemptu mundi ....... s. LIX

De ma[n]suetudine .............. s. LXII

Contra impedientes verbum Dei ...... s. s. LXII

De subversione divitiarum .......... s. LXIII

Contra nolentes dare pauperibus...... s. s. LXVI

De honore parentum .............. s. LXX

Contra non corrigentes pueros........ s. LXXII

De castitate................... s. LXXVI

Contra defraudantes mortuos ........ s. LXXXV

Contra servos linguosos ........... s. LXXXXII

Contra luxuriose viventes............ LXXXXIIII

Contranon substentantesparentessuos. s. LXXXXV

Contra non observantes fidem in matrimonio ..................

Contra blasphemantes Deum............

s. LXXXXI 
Ex. de comite quodam Campanie

Ex. de Johanne elemosinario.... .

Ex. de domina quadam nobili ...

Ex. de Constantino imperatore. .

Ex. de vendente solem .........

Ex. de sole . ...............

Ex. de sole vendito. ...........

Ex. de quodam faciente nutus equivocos.................

Ex. de domina quadam.........

Ex. de quodam obsesso.........

Ex. de Moyse ..............

Ex. de moniali quadam

Ex. de quodam inaniter gloriante
De operibus pietatis..............

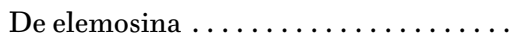

De elemosina ....................

De cruce ......................

Contra accipientes per calumpiam ....

Contra $[\ldots . . .$.$] per calumpiam .........$

De diversis modis [nocendi $]. . . \ldots \ldots$.

Contra adulantes blande............

Contra ancillas inhonestas ..........

De terrore dyaboli in confessionem....

Contra dantes filias suas usurariis .....

Contra fatuas mulieres se ostendantes in vilicos

Contra inaniter gloriantes de nobilitate propria .................... s. XLIX

s. XLIX

s. XLIX

s. LVII

s. LXI

s. LXI

s. LXII

s. LXIIII

s. LXXII

CVIII

CXV

CXVI

XXXV

$\mathbf{U}^{69}$

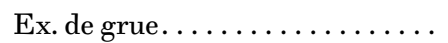

Contra luxuriosos sacerdotes .........

s. IIII

Ex. de muliere .............

Contra amicos infideles ${ }^{70}$ carnales ....

s. LV

Ex. de cruce ostensum $[s i c]$ cuidam religioso $\ldots \ldots \ldots \ldots \ldots \ldots \ldots \ldots$

s. LVIII

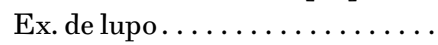

Contra capientes per calumpniam.....

s. LXI

Ex. de bubone ..............

Contra non exequentes efficaciter.....

Ex. de rustico

Contra non servantes festa ..........

s. [...]

Ex. de muliere avara ..........

Contra non agentes elemosinas .......

Ex. de fure quodam. ..........

Contra non corrigentes pueros pro-

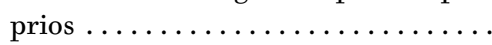

s. LXVI

s. $[\ldots]$

Ex. de juvene nobili.

De mortis meditatione . ............

s. $\operatorname{LX}[\ldots]$

s.LX $[\ldots]$

Contra impatientes ...............

s. LX $[\ldots]$

Contra non facientes penitentiam.....

s. LXX[...]

Ex. de puero fure.............

Ex. de puero quinquenni .......

Ex. de vulpe

Contra blasphemantes et jurantes et perjurantes................... s. LXXXXVI

Contra luxuriam aliaque peccata...... s. LXXXXV

Ex. de judice quodam

Contra accipientes in judiciis

s. CXV

Explicit tabula utilis et facilis ad inveniendum omnia exempla hic posita et contenta.

Deo gratias.

69. À partir de ce point, la reliure mord sur le bord de la page, rendant les numéros de sermon très difficilement lisibles.

70. infideles] suscrit. 
3 B

Édition de la «tabula exemplorum 》 du ms. Bologne, Collegio di Spagna, 56, fol. 204v-205

[fol. 204vb] Tabula exemplorum precedentium sermonum.

$\mathbf{A}$

De duobus fratribus servientibus infirmis .

Opus misericordie ............ 38

De advocato infideli ................

Avaritia ................... 46

De sancto Martino . . . . . . . . . . . . . .

Opus misericordie ............ 49

De sancto Francisco . . . . . . . . . . . . . . .

Opus misericordie ............. 49

De sancto Antonio ..................

Opus misericordie ............. 49

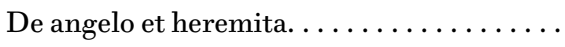

Fetor peccati............... 56

De sancto Martino ..................

De Karolo Magno ....................

De Alexandro .....................

De panthera.......................

De avaro........................

Virtus crucis ................ 59

Contra non audientes predicationem.. 61

Contra adulatores.............. 61

Contra nolentes audire sermonem.... 62

Contra avaritia ................. 66

De Paulo abbate ..................

De marito et uxore ..................

De matre silvestri caprioli .............

De quodam sapiente................

Contra ociosos ............... 68

Contra non honorantes invicem..... 70

De obedientia ad parentes......... 95

De mortis consideratione ........ 85

E

De leone.........................

De quodam peregrino................

De quodam rege...$\ldots \ldots \ldots \ldots \ldots \ldots$

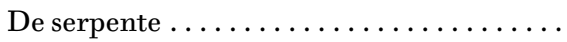

De vespertilione $\ldots \ldots \ldots \ldots \ldots \ldots \ldots \ldots$

De Philipo re[ge] . ..................

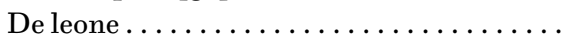

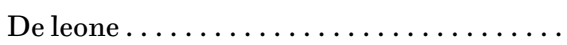

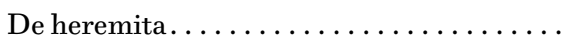

De rege habente III filias . . . . . . . . . . . .

De Herode .......................

De demone.......................

Contra gravantes pauperes subditos .. 40

De contemplatione............ 61

Contra personarum acceptionem..... 61

Contra adulatores............. 61

Contra opprimentes pauperes ...... 62

Contra sibi nocentes............ 62

Contra nocentes vicinis .......... 66

De opere misericordie ........... 66

Contra occiosos $[s i c] \ldots \ldots \ldots \ldots \ldots 68$

De honore parentum ............ 70

De inordinato amore ............ 72

Contra fatuas mulieres .......... 72

De sancta Thecla................ De castitate............... 76

De famulis scolarium .............. De infidelitate servientium ........ 91

De beati $[$ sic $]$ Bernardo ............. De contemptu mundi........... 94

De servo ................... De servo pigro.............. 91

De Renardo ................... Contra recidivantes ........... 95

De feneratore.......................... Contra usurarios.... $116^{71}$

71. 116] 106 ante corr. 
De ligno arido .....................

De Scipione Affricano .................

De milite quodam ..................

De imperatore Troiano $[s i c] \ldots \ldots \ldots \ldots$

De milite quodam ..................

De Socrate philosopho ...............

[fol. 205a]

De milite

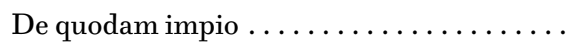

De virgine $\ldots \ldots \ldots \ldots \ldots \ldots \ldots \ldots$

De milite $\ldots . \ldots \ldots \ldots \ldots \ldots \ldots \ldots$. . . .

De divite quodam $\ldots \ldots \ldots \ldots \ldots \ldots \ldots$.

De viro sancto $\ldots \ldots \ldots \ldots \ldots \ldots \ldots \ldots$

De malo filio .....................

De cyconia ......................

De milite ......................

\section{O}

De comite Campanie ................

De Johanne Elemosinario ..............

De domina nobili. ..................

De imperatore Constantino.............

De quodam vendente solem .............

De sole .........................

De quodam faciente nutus equivocos ......

De quadam domina. . . . . . . . . . . . . .

De quodam obsesso.................

\section{U}

De grue........................

De muliere .....................

De cruce ostensa cuidam relig[ioso $]. . . .$. .

De luppo $[s i c] \ldots \ldots \ldots \ldots \ldots \ldots \ldots \ldots$

De bubone ......................

De muliere avara ..................

De rustico.......................

De fure...$\ldots \ldots \ldots \ldots \ldots \ldots \ldots$

De juvene nobili. ..................

De muliere litigiosa.................
De obbedientia $[s i c] \ldots \ldots \ldots \ldots \ldots . \ldots 38$

Castitas .................... 46

De cruce et contemptu mundi....... 59

De mansuetudine ............... 62

Contra impedientes verbum Dei..... . 62

De voluntaria paupertate ........ 63

Contra nolentes dare pauperibus .... . 66

Contra non honorantes parentes ..... 70

De castitate...................... 76

Pro suffragiis mortuorum .......... 85

Contra linguosos .............. 92

Contra luxuriam.............. 94

Contra non sustentantes parentes .... 95

Contra uxoratos malos........... 70

Contra blasphemiam .......... $91^{72}$

De misericordia $\ldots \ldots \ldots \ldots \ldots \ldots .49$

.................... 49

Elemosina................. 49

De cruce.................. 57

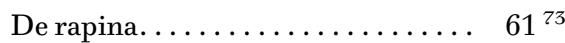

........................ 62

De fraude................. 64

Contra inhonestas ancillas ........ 72

De terrore confessionis .......... 108

Contra luxuriosos sacerdotes ....... 4

Contra amicos carnales........... 55

.......................... 58

Contra rapinam .............. 61

Contra negligentes............. 62

Contra avaros ................. 66

Contra non observantes festa ....... 66

Contra negligentes correctores...... 62

De morte.......................... 85

Contra impacientes ........... 91

72. Suivent deux lignes barrées «De Moyse - Contra dantes filias usurariis - 115. / De moniali. »

73. 61] le renvoi est répété à la ligne suivante, sans correspondre à un exemplum. Il semble que le scribe ait ici omis une entrée de la table, tout en copiant son renvoi. 
[fol. 205b]

De puero fure.......................... Contra impenitentes 95

De puero quinqueni ............... Contra blasphemantes.......... 96

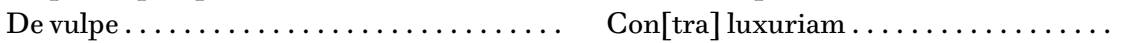


La table des distinctions et le début de la table des exempla, classées selon les voyelles.

Paris, Bibl. nat. Fr., lat. 16480, fol. 175v-176. (Cl. BNF.)

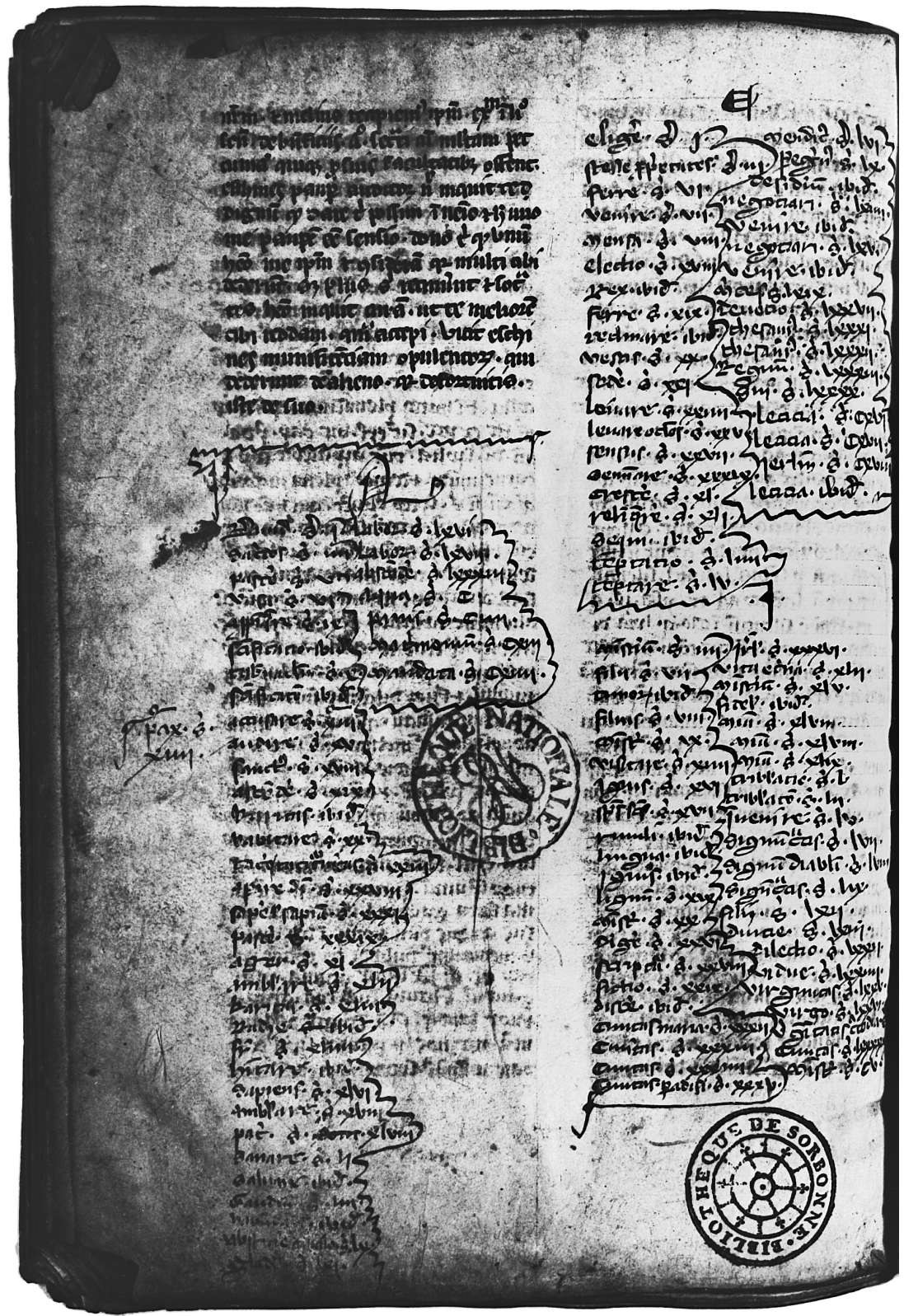




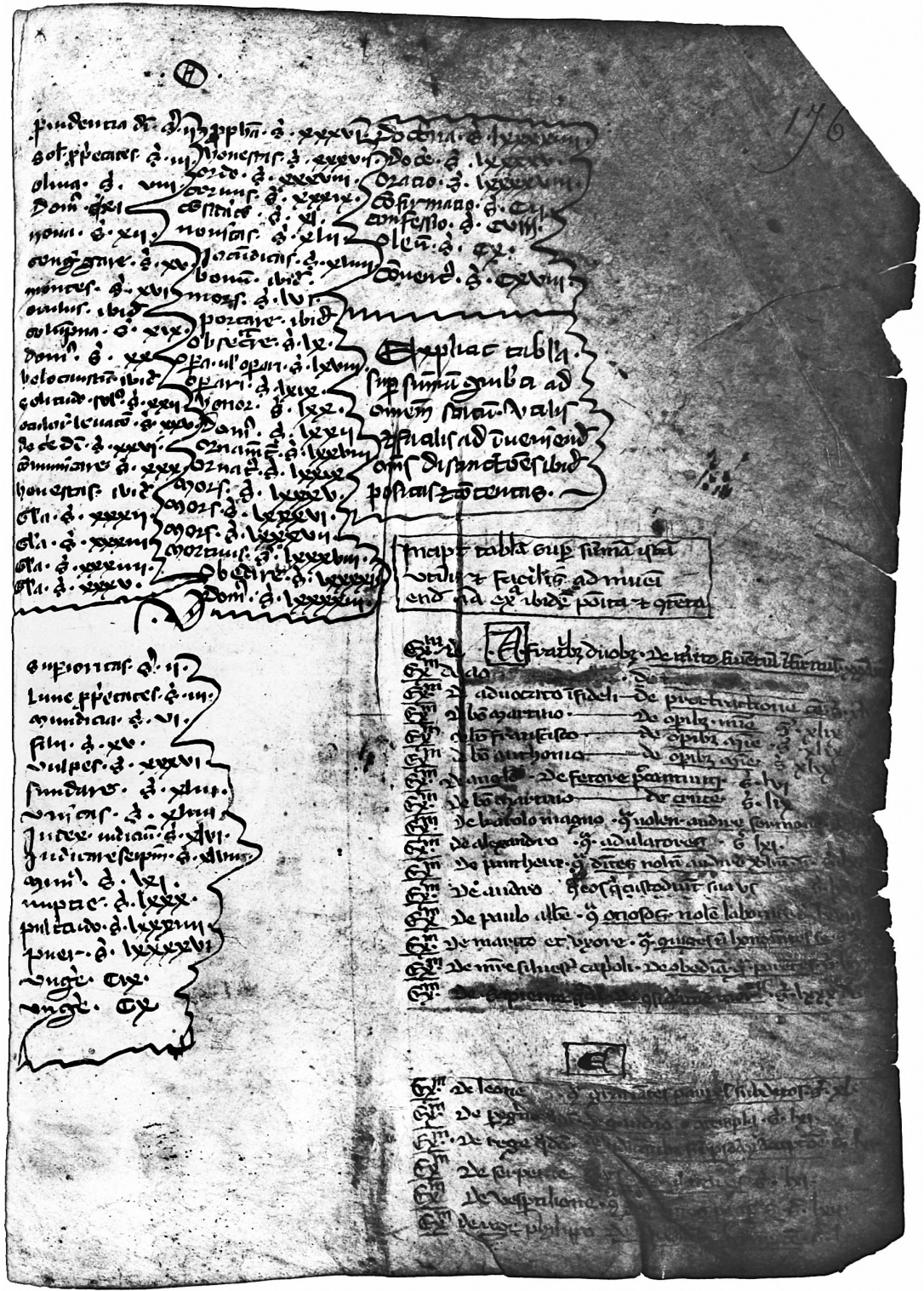




\section{B}

La fin de la table des distinctions (ordre alphabétique traditionnel) et le début de la table des exempla (selon les voyelles).

Bologne, Collegio di Spagna, ms. 56, fol. 204v.

(Progetto Irnerio. Cirsfid, Università degli Studi di Bologna, avec le soutien de la Fondazione CARISBo ; reprod. autorisée.)

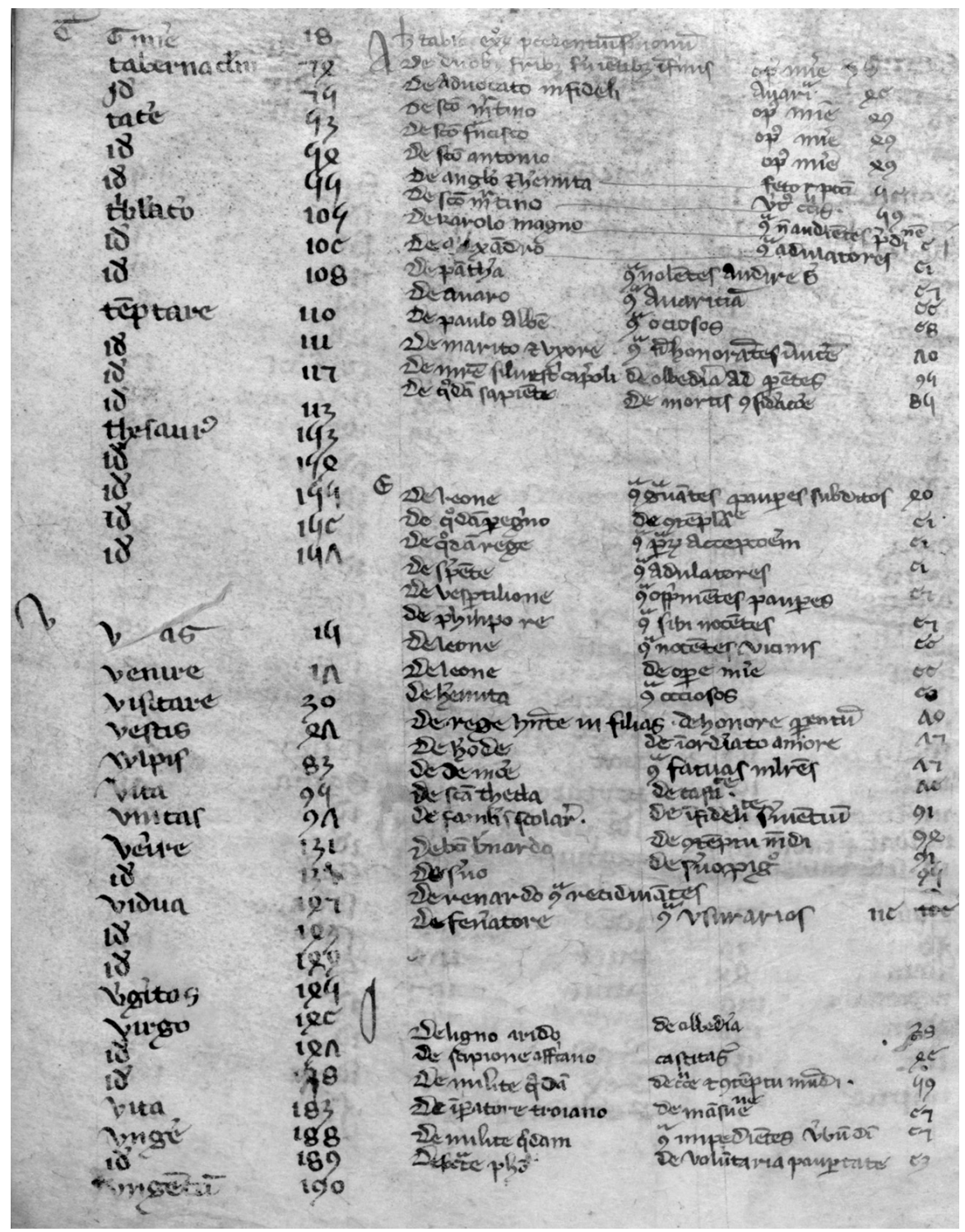

